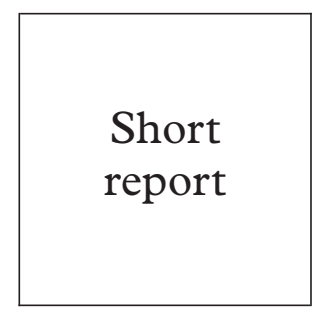

Respiratory Virus London, UK

$M$ Saville

D Brown ${ }^{\star}$

MDA Evaluation Group CPHL

C Burgess

K Perry

HIV/Genitourinary

Medicine Services, St

Stephen's Centre,

Chelsea and

Westminster Hospital

$S$ Barton ${ }^{\star}$

Department of STDs, University College

London, Mortimer

F Cowan*

Instituo di

Microbiologia,

Universita di Padova,

Padova, Italy

G Palu

C Mengoli

${ }^{\star}$ On behalf of the MSSVD Special Interest Group on Genital Herpes.

Correspondence to: Dr Brown, PHLS, Enteric and Respiratory Virus Laboratory, PHLS,

Colindale Avenue, London, UK

dbrown@)cphl.demon.co.uk

Accepted for publication 10 August 2000
Enteric and Laboratory, CPHL,

Market Centre

\section{An evaluation of near patient tests for detecting herpes simplex virus type- 2 antibody}

\author{
Melanie Saville, David Brown, Christine Burgess, Keith Perry, Simon Barton, Frances \\ Cowan, Giorgio Palu, Carlo Mengoli
}

\begin{abstract}
Objectives: To examine the "in use" test characteristics of the POCkit "near patient" HSV-2 rapid test for the detection of HSV-2 IgG antibodies for use in the clinic. This test relies on a visual interpretation of the result.
\end{abstract}

Methods: 2093 serum samples, 229 from UK and 919 from Italian genitourinary medicine clinic patients and 945 from obstetric and gynaecology clinic patients in Italy were tested. Tests were carried out according to manufacturers' protocol in the United Kingdom and Italy. Three readers independently recorded a score for each test carried out and the results were compared.

Results: In the UK study, the three readers disagreed on the result on $5.2 \%$ of tests. In the Italian study, there was disagreement in $10.2 \%$ of tests.

Conclusions: This study has demonstrated a problem in the subjective nature of the interpretation of the POCkit HSV-2 test. It highlights the need for adequate training of clinic staff and the need for clinics to adopt policies of quality assurance and ongoing monitoring which will ensure the validity and accuracy of this clinic based test. (Sex Transm Inf 2000;76:381-382)

Keywords: evaluation; herpes simplex virus; POCkit HSV-2 test
Herpes simplex virus type 2 (HSV-2) is the principal cause of genital herpes. Traditionally, infection has been diagnosed by isolation of virus or viral antigen detection directly from genital lesions. However, this approach is impractical for diagnosing infection in the absence of a lesional recurrence or for diagnosing infection in people with asymptomatic infection. ${ }^{1}$ Recently, a number of commercial assays which can detect antibodies to HSV-2 have been developed for use in diagnostic serology laboratories. In addition, POCkit HSV-2 rapid test has been developed as a near patient test designed for use in the clinic. The POCkit test detects HSV-2 type specific IgG antibodies in either whole blood or serum. Interpretation of the test result relies on visualising a pink/red coloured spot in the test device. Early evaluation of this test compared with western blot analysis on 87 specimens found that the POCkit HSV-2 rapid test had a sensitivity of $96 \%$ and a specificity of $97 \% .^{2}$ In this report we present results of two separate evaluations of the POCkit test, one conducted in London and the other in Italy, which examine the "in use" test characteristics.
Table 1 Scoring system used to read the POCkit HSV 2 rapid test*

\begin{tabular}{lll}
\hline Test spot observation & Test score & HSV-2 antibody status \\
\hline No red/pink test spot observed & 0 & Negative \\
Some red/pink colour visible in the test but not as a clear spot & 1 & Ambiguous \\
Very weak, but definite test spot & 2 & Positive \\
A medium reaction in test spot & 3 & Positive \\
A strongly reactive specimen & 4 & Positive \\
\hline
\end{tabular}

${ }^{\star} \mathrm{A}$ pink/red control spot has to be present in each test device to make the test valid.

\section{Subjects, methods, and results}

UK

In all, 229 serum samples were collected from selected genitourinary medicine clinic patients through the MSSVD Advisory Panel on genital herpes.

ITALY

A total of 919 serum samples were collected in an STD clinic and 945 serum samples were collected in an obstetric and gynaecology clinic.

In both cases the tests were carried out according to the manufacturers' protocol. Owing to the subjective nature of the test, a scoring scheme already developed for evaluation of other near patient serology tests ${ }^{3}$ (table 1) was applied to the POCkit HSV rapid test. From this, three independent readers recorded a score for each of the tests.

In the UK study, the three readers disagreed on the result in 12 out of 229 samples (5.2\%). In two of these 12 samples, a positive/negative and ambiguous reading was given by each of the different readers of the same specimen, and in the remainder at least two different test results were given for each specimen. In the Italian study there was disagreement about 192 samples $(10.2 \%)$. The majority, 182 , were read as ambiguous (test score 1) by at least one of the readers.

\section{Discussion}

This study has demonstrated a problem in the subjective nature of the interpretation of the POCkit HSV-2 test. This caused difficulty with the interpretation of the result even in the hands of highly experienced laboratory staff. Of note, this test is designed to be used in clinics, pharmacies, and by the general public, 
when it will often be carried out by nonlaboratory trained staff. It is clearly important that these staff receive adequate training in the interpretation of these tests, with ongoing evaluation to ensure consensus of interpretation. In this study some patients undergoing testing could have received either a false positive or false negative HSV-2 antibody result with likely unfortunate consequences. ${ }^{4}$ This assay is now sold for home testing directly to patients (wellbeing met), which may lead to further diagnostic confusion.

At the time of this evaluation, the POCkit HSV-2 rapid test did not include the option of an "ambiguous" result. However, the manufacturer of the test kit, Diagnology, have responded to this problem by clarifying the interpretation criteria particularly to cases where the test spot is very faint, as being an "indeterminate" result, cautioning users about the possibility of overinterpretation of these faint results. A further serological test 4-6 weeks later is recommended. This response is welcomed and must be publicised.
Importantly, extensive quality assurance schemes exist within laboratories to ensure that test results are reproducible. With the likely increase in near patient test kits, it is essential that large scale evaluations in laboratories are undertaken before clinic use and that clinics adopt policies of quality assurance and ongoing monitoring which will ensure the validity and accuracy of clinic based testing. In addition, it should be established that similar performance characteristics of the near patient test can be guaranteed in non-laboratory settings before they are widely introduced.

1 Munday PE, Vuddamalay J, Slomka MJ, et al. Role of type specific herpes simplex virus serology in the diagnosis and management of genital herpes. Sex Transm Inf 1998;74:175-8.

2 Ashley R, Eagleton M. Evaluation of a novel point of care test for antibodies to herpes simplex virus type- 2 . Sex test for antibodies to herpe

3 Giles RE, Gollapalli M, Perry KR, et al. Thirteen anti-HIV screening simple/rapid test devices. MDA/98/27. Medical Devices Agency, 1998

4 Field PR, Ho DWT, Irving WL, et al. The reliability of seroogical tests for the diagnosis of genital herpes: a critique. Pathology 1993;25:175-9. 